Commun. Korean Math. Soc. 22 (2007), No. 4, pp. 503-508

\title{
A METHOD TO MAKE BCK-ALGEBRAS
}

\author{
Young Bae Jun, Kyoung Ja Lee, and Chul Hwan Park
}

\begin{abstract}
Using the notion of posets, a method to make BCK-algebras is considered. We show that if a poset has the least element, then the induced BCK-algebra is bounded.
\end{abstract}

\section{Introduction and basic results on BCK-algebras}

BCK-algebras entered into mathematics in 1966 through the work of Imai and Iséki [3], and have been applied to many branches of mathematics, such as group theory, functional analysis, probability theory and topology. Such algebras generalize Boolean rings as well as Boolean $D$-posets (= $M V$-algebras). Founding new BCK-algebras is important in studying BCK-algebras and related algebraic structures. A way to make a new BCK-algebra from old is established by Abujabal [1]. Jun et al. [7] gave a method to make a BCKalgebra from a poset and an upper set. Hao [2] gave a method for constructing a proper BCC-algebra by the extension of a BCK-algebra with a small atom. Iséki [6] gave a method to make a BCI-algebra by using a group and a BCKalgebra. In this paper, we give a method to make a BCK-algebra by using a poset. We show that if a poset has the least element, then the induced BCK-algebra is bounded.

We first display basic concepts on BCK-algebras. By a $B C K$-algebra we mean an algebra $(X ; *, 0)$ of type $(2,0)$ satisfying the axioms:

(a1) $(\forall x, y, z \in X)(((x * y) *(x * z)) *(z * y)=0)$,

(a2) $(\forall x, y \in X)((x *(x * y)) * y=0)$,

(a3) $(\forall x \in X)(x * x=0,0 * x=0)$,

(a4) $(\forall x, y \in X)(x * y=0, y * x=0 \Rightarrow x=y)$.

We can define a partial ordering $\leq$ by $x \leq y$ if and only if $x * y=0$. A BCKalgebra $X$ is said to be bounded if there exists the bound 1 such that $x \leq 1$ for all $x \in X$. A mapping $f: X \rightarrow Y$ of BCK-algebras is called a homomorphism if $f(x * y)=f(x) * f(y)$ for all $x, y \in X$.

Received July 9, 2007.

2000 Mathematics Subject Classification. 06F35, 06A06, 03G25.

Key words and phrases. (irreducible) ideal, order system, decreasing subset. 
In any BCK-algebra $X$, the following hold:

(b1) $(\forall x \in X)(x * 0=x)$,

(b2) $(\forall x, y, z \in X)((x * y) * z=(x * z) * y)$,

(b3) $(\forall x, y, z \in X)((x * z) *(y * z) \leq x * y)$,

(b4) $(\forall x, y, z \in X)(x \leq y \Rightarrow x * z \leq y * z, z * y \leq z * x)$.

\section{Making BCK-algebras}

In what follows let $X$ denote a BCK-algebra unless otherwise specified. The following definition is well-known.

Definition 2.1. A subset $A$ of $X$ is called an ideal of $X$ if it satisfies:

(c1) $0 \in A$,

(c2) $(\forall x \in A)(\forall y \in X)(y * x \in A \Rightarrow y \in A)$.

Note that every ideal $A$ of $X$ satisfies:

$$
(\forall x \in A)(\forall y \in X)(y \leq x \Rightarrow y \in A) \text {. }
$$

The set of all ideals of $X$ is denoted by $\operatorname{Id}(X)$. It is known that $\operatorname{Id}(X)$ is an infinitely distributive lattice (see [10]). If $A$ is a nonempty subset of $X$, then the ideal of $X$ generated by $A$, in symbol $\langle A\rangle$, is the set (see [4, Theorem 3])

$$
\langle A\rangle=\left\{\begin{array}{l|l}
x \in X & \begin{array}{l}
\left(\cdots\left(\left(x * a_{0}\right) * a_{1}\right) * \cdots\right) * a_{n}=0 \\
\text { for some } a_{0}, a_{1}, \ldots, a_{n} \in A
\end{array}
\end{array}\right\} .
$$

Definition 2.2. An ideal $A$ of $X$ is said to be irreducible (see [5]) if it satisfies:

$$
(\forall B, C \in I d(X))(A=B \cap C \Rightarrow A=B \text { or } A=C) .
$$

Denote by $I I d(X)$ the set of all irreducible ideals of $X$.

Lemma 2.3 ([5, Theorem 2]). Let $A \in I d(X)$. If $A$ is irreducible, then

$$
(\forall a, b \in X \backslash A)(\exists c \in X \backslash A)(c \leq a, c \leq b) .
$$

Lemma 2.4. Let $A \in I d(X)$. Then the following are equivalent:

(i) $A$ is irreducible.

(ii) $(\forall a, b \in X \backslash A)(\exists c \in X \backslash A)(c * a, c * b \in A)$.

Proof. (i) $\Rightarrow$ (ii) This follows from Lemma 2.3.

(ii) $\Rightarrow$ (i) Let $B, C \in I d(X)$ be such that $A=B \cap C$. Assume that $A \neq B$ and $A \neq C$. Then there exist $b \in B \backslash A$ and $c \in C \backslash A$. It follows from (ii) that there exists $d \in X \backslash A$ such that $d * b \in A$ and $d * c \in A$. From $b \in B \backslash A \subseteq B$ and $d * b \in A=B \cap C \subseteq B$, we have $d \in B$ since $B \in I d(X)$. Similarly, $d \in C$, and so $d \in B \cap C=A$. This is a contradiction. Therefore $A$ is an irreducible ideal of $X$.

Definition 2.5. A subset $I$ of $X$ is called an order system of $X$ if it satisfies: 
(c3) $I$ is an upper set, that is, $I$ satisfies:

$$
(\forall x \in X)(\forall y \in I)(y \leq x \Rightarrow x \in I),
$$

(c4) $(\forall x, y \in I)(\exists z \in I)(z \leq x, z \leq y)$.

Denote by $O s(X)$ the set of all order systems of $X$.

Theorem 2.6. Let $A \in I d(X)$ and $I \in O s(X)$. If $A$ and $I$ are disjoint, then there exists an irreducible ideal $B$ of $X$ such that $A \subseteq B$ and $B \cap I=\emptyset$.

Proof. Let

$$
\mathscr{X}:=\{H \in I d(X) \mid A \subseteq H, H \cap I=\emptyset\} .
$$

Then $\mathscr{X} \neq \emptyset$ since $A \in \mathscr{X}$. Obviously, the union of a chain of elements of $\mathscr{X}$ is contained in $\mathscr{X}$. Using Zorn's lemma, $\mathscr{X}$ has a maximal element, say $B$. Let $a, b \in X \backslash B$ and consider ideals $\langle B \cup\{a\}\rangle$ and $\langle B \cup\{b\}\rangle$ generated by $B \cup\{a\}$ and $B \cup\{b\}$, respectively. Clearly $B \subseteq\langle B \cup\{a\}\rangle \cap\langle B \cup\{b\}\rangle$, and so $\langle B \cup\{a\}\rangle \cap I \neq \emptyset$ and $\langle B \cup\{b\}\rangle \cap I \neq \emptyset$. If not, then $\langle B \cup\{a\}\rangle \in \mathscr{X}$ or $\langle B \cup\{b\}\rangle \in \mathscr{X}$. This contradicts to the fact that $B$ is a maximal element of $\mathscr{X}$. Hence there exist $x, y \in I$ such that $x \in\langle B \cup\{a\}\rangle$ and $y \in\langle B \cup\{b\}\rangle$. It follows that $x * a \in B$ and $y * b \in B$. Since $I$ is an order system of $X$, there exists $z \in I$, and so $z \in X \backslash B$, such that $z \leq x$ and $z \leq y$. It follows from (b4) that $z * a \leq x * a$ and $z * b \leq y * b$ so from (2.1) that $z * a \in B$ and $z * b \in B$. We conclude from Lemma 2.4 that $B$ is irreducible.

Let $(P, \leq)$ be a poset. For any $a \in P$, we put

$$
(a]:=\{x \in P \mid x \leq a\} \text {. }
$$

For any $X \subseteq P$, we put

$$
(X]:=\bigcup_{a \in X}(a] .
$$

For any $X \subseteq P$, if $X=(X]$ then we say that $X$ is a decreasing subset of $P$. Denote by $\mathscr{D}(P)$ the family of all decreasing subsets of $P$.

Theorem 2.7. If $(P, \leq)$ is a poset, then $(\mathscr{D}(P), \odot, P)$ is a BCK-algebra where the operation $\odot$ is defined as follows:

$$
(\forall X, Y \in \mathscr{D}(P))(X \odot Y=\{a \in P \mid(a] \cap Y \subseteq X\}) .
$$

Proof. The proof is routine.

Example 2.8. Let $P=\{1,2,3\}$ be a poset with the following Hasse diagram:

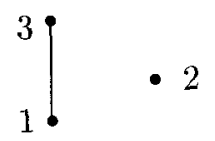


Then $(1]=\{1\},(2]=\{2\}$ and $(3]=\{1,3\}$. It is routine to verify that

$$
\mathscr{D}(P)=\{P,\{1,2\},\{1,3\},\{1\},\{2\}\},
$$

and it is a BCK-algebra with the following Cayley table:

\begin{tabular}{c|ccccc}
$\odot$ & $P$ & $\{1,2\}$ & $\{1,3\}$ & $\{1\}$ & $\{2\}$ \\
\hline$P$ & $P$ & $P$ & $P$ & $P$ & $P$ \\
$\{1,2\}$ & $\{1,2\}$ & $P$ & $\{1,2\}$ & $P$ & $P$ \\
$\{1,3\}$ & $\{1,3\}$ & $\{1,3\}$ & $P$ & $P$ & $\{1,3\}$ \\
$\{1\}$ & $\{1\}$ & $\{1,3\}$ & $\{1,2\}$ & $P$ & $\{1,3\}$ \\
$\{2\}$ & $\{2\}$ & $\{2\}$ & $\{2\}$ & $\{2\}$ & $P$
\end{tabular}

Example 2.9. Consider the letter $\mathbf{N}$ poset (see [9]):

$$
P:=\{a, b, c, d\} \text { and } \leq:=\{(a, a),(b, b),(c, c),(d, d),(a, c),(b, c),(b, d)\} .
$$

The Hasse diagram for this poset is as follows:

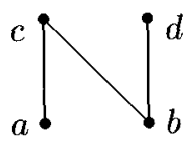

Then $(a]=\{a\},(b]=\{b\},(c]=\{a, b, c\}$ and $(d]=\{b, d\}$. Decreasing subsets of $P$ are $\{a\},\{b\},\{a, b\},\{b, d\},\{a, b, c\},\{a, b, d\}$ and $P$, that is,

$$
\mathscr{D}(P)=\{P,\{a\},\{b\},\{a, b\},\{b, d\},\{a, b, c\},\{a, b, d\}\} .
$$

Using (2.8), we have the following Cayley table:

\begin{tabular}{c|ccccccc}
$\odot$ & $P$ & $\{a\}$ & $\{b\}$ & $\{a, b\}$ & $\{b, d\}$ & $\{a, b, c\}$ & $\{a, b, d\}$ \\
\hline$P$ & $P$ & $P$ & $P$ & $P$ & $P$ & $P$ & $P$ \\
$\{a\}$ & $\{a\}$ & $P$ & $\{a\}$ & $\{a\}$ & $\{a\}$ & $\{a\}$ & $\{a\}$ \\
$\{b\}$ & $\{b\}$ & $\{b, d\}$ & $P$ & $\{b, d\}$ & $\{a, b, c\}$ & $\{b, d\}$ & $\{b\}$ \\
$\{a, b\}$ & $\{a, b\}$ & $P$ & $P$ & $P$ & $\{a, b, c\}$ & $\{a, b, d\}$ & $\{a, b, c\}$ \\
$\{b, d\}$ & $\{b, d\}$ & $\{b, d\}$ & $P$ & $\{b, d\}$ & $P$ & $\{b, d\}$ & $\{b, d\}$ \\
$\{a, b, c\}$ & $\{a, b, c\}$ & $P$ & $P$ & $P$ & $P$ & $P$ & $\{a, b, c\}$ \\
$\{a, b, d\}$ & $\{a, b, d\}$ & $P$ & $P$ & $P$ & $P$ & $\{a, b, d\}$ & $P$
\end{tabular}

Example 2.10. Let $P=\{0,1,2\}$ be a poset with the following Hasse diagram:

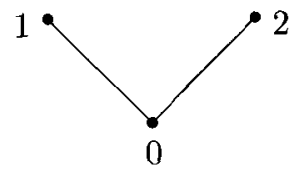

Then $(0]=\{0\},(1]=\{0,1\}$ and $(2]=\{0,2\}$. We know that

$$
\mathscr{D}(P)=\{P,\{0,1\},\{0,2\},\{0\}\},
$$


and it is a bounded BCK-algebra with the following Cayley table:

\begin{tabular}{c|cccc}
$\odot$ & $P$ & $\{0,1\}$ & $\{0,2\}$ & $\{0\}$ \\
\hline$P$ & $P$ & $P$ & $P$ & $P$ \\
$\{0,1\}$ & $\{0,1\}$ & $P$ & $\{0,1\}$ & $P$ \\
$\{0,2\}$ & $\{0,2\}$ & $\{0,2\}$ & $P$ & $P$ \\
$\{0\}$ & $\{0\}$ & $\{0,2\}$ & $\{0,1\}$ & $P$
\end{tabular}

Lemma 2.11. If $(P, \leq)$ is a poset with the least element, then every decreasing subset of $P$ contains the least element.

Proof. Let $X$ be a decreasing subset of $P$ and let $a$ be the least element of $(P, \leq)$. Then $a \in(x]$ for all $x \in P$, and so

$$
a \in \bigcup_{w \in X}(w]=(X]=X \text {. }
$$

This completes the proof.

Theorem 2.12. If $(P, \leq)$ is a poset with the least element $a$, then the induced $B C K$-algebra $(\mathscr{D}(P), \odot, P)$ is bounded with the bound $(a]$.

Proof. Since $a$ is the least element of $(P, \leq)$, we have $(a]=\{a\}$ and $a \in(w]$ for all $w \in P$. Hence $(w] \cap(a]=\{a\}$ for all $w \in P$. Now if $X \in \mathscr{D}(P)$, then $a \in X$ by Lemma 2.11 . Therefore

$$
X \odot(a]=\{y \in P \mid(y] \cap(a]=\{a\} \subseteq X\}=P,
$$

that is, $(a]$ is the bound of the induced BCK-algebra $(\mathscr{D}(P), \odot, P)$.

Let $\leq$ be an order relation on $I I d(X)$ defined by

$$
(\forall A, B \in \operatorname{IId}(X))(A \leq B \Leftrightarrow B \subseteq A) .
$$

Then $(\operatorname{Id}(X), \leq)$ is a poset, and $(\mathscr{D}(\operatorname{IId}(X)), \odot, \operatorname{Id}(X))$ is a BCK-algebra. Let $f: X \rightarrow \mathscr{D}(I I d(X))$ be a mapping defined by

$$
(\forall x \in X)(f(x)=\{A \in I I d(X) \mid x \in A\}) .
$$

Lemma 2.13. Let $A \in I I d(X)$ be such that $x * y \in A$ for every $x, y \in A$. If $B \in I I d(X)$ satisfies $B \in(A] \cap f(y)$, then $B \in f(x)$.

Proof. Let $B \in I I d(X)$ be such that $B \in(A] \cap f(y)$. Then $B \in(A]$ and $B \in f(y)$, and so $B \leq A$, i.e., $A \subseteq B$ and $y \in B$. Since $x * y \in A \subseteq B$, it follows from (c2) that $x \in \bar{B}$ so that $B \in \bar{\epsilon}(x)$.

Theorem 2.14. The mapping $f: X \rightarrow \mathscr{D}(\operatorname{IId}(X))$ which is established in (2.11) is a homomorphism.

Proof. Let $A \in f(x * y)$ for all $x, y \in X$. Then $A \in I I d(X)$ and $x * y \in A$. Thus if $B \in I I d(X)$ and $B \in(A] \cap f(y)$, then $B \in f(x)$ by Lemma 2.13 , and so $A \in f(x) \odot f(y)$. Conversely, assume that $A \in I I d(X)$ and $(A] \cap f(y) \subseteq f(x)$ for all $x, y \in X$. If $x * y \notin A$, then consider an ideal $A_{y}:=\langle A \cup\{y\}\rangle$. Since 
$x \notin A_{y}$, there exists $B \in I I d(X)$ such that $A \subseteq B, y \in B$ and $x \notin B$. Hence $A \notin f(x) \odot f(y)$, which is a contradiction. Therefore $x * y \in A$ and $A \in f(x * y)$. Consequently, $f$ is a homomorphism.

Theorem 2.15. Let $f: X \rightarrow \mathscr{D}(I I d(X))$ be the mapping which is established in (2.11). Then

$$
f(X):=\{f(x) \mid x \in X\}
$$

is a subalgebra of $\mathscr{D}(I I d(X))$, which is isomorphic to $X$.

Proof. Clearly, $f(X)$ is a subalgebra of $\mathscr{D}(\operatorname{IId}(X))$. It is also clear that the mapping $f: X \rightarrow \mathscr{D}(\operatorname{IId}(X))$ is injective. Since $f$ is a homomorphism, it follows that $X$ and $f(X)$ are isomorphic.

\section{References}

[1] H. A. S. Abujabal, A relative one-point union of BCK-algebras, Math. Japonica 45 (1997), no. 1, 103-111.

[2] J. Hao, Ideal theory of BCC-algebras, Scientiae Mathematicae 1 (1998), no. 3, 373-381.

[3] Y. Imai and K. Iséki, On axiom systems of propositional calculi, Proc. Jpn. Acad. 42 (1966), 19-21.

[4] K. Iséki, On ideals in BCK-algebras, Math. Seminar Notes 3 (1975), 1-12.

[5] — On some ideals in BCK-algebras, Math. Seminar Notes 3 (1975), 65-70.

[6] Some examples of BCI-algebras, Math. Seminar Notes 8 (1980), 237-240.

[7] Y. B. Jun, J. Y. Kim, and H. S. Kim, On Q-upper algebras, Order 22 (2005), 191-200.

[8] J. Meng and Y. B. Jun, BCK-algebras, Kyungmoon Sa Co. Korea, 1994.

[9] J. Neggers and H. S. Kim, Basic Posets, World Scientific Publishing Co. 1998.

[10] M. Palasiński, On ideals and congruence lattices of BCK-algebras, Math. Seminar Notes $\mathbf{9}(1981), 441-443$.

Young BAE Jun

Department of Mathematics Education (AND RINS)

Gyeongsang National University

Chinju 660-701, Korea

E-mail address: skywine@gmail.com

KYOUNG JA LEE

SCHOOL OF General Education

KOOKMIN UNIVERSITY

SEOUL 136-702, KoREA

E-mail address: lsj1109@kookmin.ac.kr

Chul HWan PARK

DePaRTMENT OF MATHEMATICS

UNIVERSITY OF ULSAN

Ulsan 680-749, Korea

E-mail address: skyrosemary@gmail.com 